\title{
Upper Gastrointestinal Bleeding
}

\author{
Vihas Patel, $M D^{1}$ Jeffrey Nicastro, $M^{2}$ \\ ${ }^{1}$ Department of Surgery, Long Island Jewish Medical Center, \\ Northwell Health, New Hyde Park, NY \\ 2 Department of Surgery, Western Connecticut Health Network, \\ Connecticut
}

\begin{abstract}
Address for correspondence Vihas Patel, MD, Department of Surgery, Long Island Jewish Medical Center, Northwell Health, New Hyde Park, New York (e-mail: vpatel13@northwell.edu).
\end{abstract}

Clin Colon Rectal Surg 2020;33:42-44.

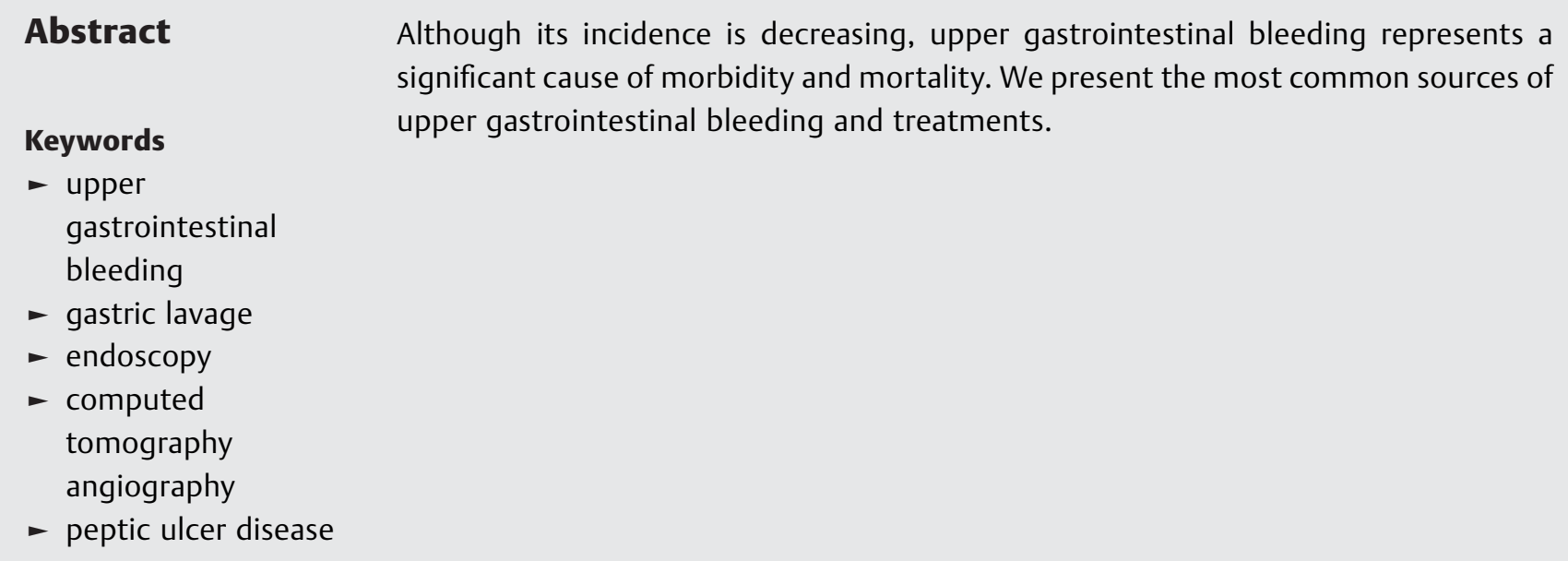

\section{Epidemiology}

The prevalence of upper gastrointestinal bleeding (UGIB) varies with geography, ranging between 37 and 172 cases per 100,000 , in part due to differences in nonsteroidal anti-inflammatory drug use, Helicobacter pylori colonization, and proton pump inhibitors (PPI). It is more common in the elderly, in men, and in those with multiple comorbid conditions. ${ }^{1-4}$ Lower socioeconomic status has also been linked with increased incidence and severity of UGIB. $^{1}$

Nevertheless, the incidence of acute UGIB over the last two decades has been declining. ${ }^{1,2}$ This marked decline likely reflects improved peptic ulcer disease (PUD) management with the use of PPI and antimicrobial therapy. While overall incidence of UGIB has declined, overall mortality has remained unchanged. Published mortality rates, secondary to UGIB, range from 5 to $14 \%{ }^{1-3}$ Risk of mortality increases markedly with age such that patients over 75 years of age have a six-fold increased rate of death. ${ }^{1}$ Death in this population does not stem directly from blood loss alone, but rather is due to diminished physiologic reserve in the presence of multiple comorbidities. ${ }^{1-3}$

Issue Theme Lower GI Bleeding; Guest Editor: David E. Rivadeneira, MD, MBA, FACS, FASCRS

\section{Clinical Presentation}

Regardless the etiology, there are classic clinical stigmata on presentation that are indicative of UGIB. Hematemesis, "coffee-ground" emesis, or nasogastric tube (NGT) lavage productive of bloody output are all indicators of UGIB. Melena, classically described as "tarry" stools, is also indicative of UGIB. ${ }^{3,5}$ Although hematochezia is traditionally thought of as a sign of lower GI bleed, hematochezia in the setting of hemodynamic instability that should be considered as a potential sign of a brisk UGIB. ${ }^{3}$ Patients, exhibiting signs and symptoms of UGIB, should undergo appropriate diagnostic workup and resuscitation as indicated by clinical severity. ${ }^{5}$

\section{Establishing the Diagnosis}

UGIB is not a singular diagnosis, but rather it is an inclusive term that encompasses a number of etiologies. The most common one being PUD is accounting for 28 to $67 \%$ of all cases. $^{1-4}$ Other common causes include varices, mucosal erosions, Mallory-Weiss tears, neoplasia, esophagitis, angioectasias, and Dieulafoy's lesions. ${ }^{2,3}$ In approximately, $8 \%$ of cases, the etiology may remain elusive. ${ }^{3}$ Additionally, in 
16 to $20 \%$ of cases, endoscopy may identify more than one causative diagnosis. ${ }^{1}$

Laboratory investigations: laboratory workup usually begins with a complete blood count, chemistries (CMP), and coagulation studies. It is important to note that since the patient is losing whole blood, they may not have an immediate drop in their hematocrit; it may take up to 4 to 6 hours to equilibrate. The CMP and coagulation studies can be used to look for clues to the source of bleeding (liver dysfunction and coagulopathies). It is expected that the blood urea nitrogen/creatinine ratio will be greater than 30 for patients with an upper GI bleed, as blood is digested to protein and reabsorbed in the small bowel. The protein is transported to the liver and metabolized via the urea cycle leading to increase urea production.,

Gastric lavage: prior to an endoscopy, a quick diagnostic test that can be employed in the emergency room is the nasogastric tube (NGT) lavage. This consists of instilling 200 to $300 \mathrm{~mL}$ of warm saline via a nasogastric tube and inspecting the aspirate for blood or bile. The added advantage of NGT lavage is in clearing out the stomach of debris and clots to improve endoscopic visualization. Occasionally, the aspirate remains clear despite repeated NGT lavage. This may occur in the setting of a competent pylorus, preventing sampling of duodenal effluent. Thus, clear aspirate on NGT lavage would be considered nondiagnostic in the workup of suspected UGIB. One study showed that an NGT lavage was associated with shorter time to endoscopy but did not make a difference in length of hospital stay, surgery, transfusion requirements, or mortality. ${ }^{8}$

Endoscopy: upper endoscopy or esophagogastroduodenoscopy (EGD) is considered the gold standard to diagnose UGIB. Further, EGD and can be used for therapeutic interventions. It has high a sensitivity of 92 to $98 \%$ and specificity of 30 to $100 \%$ for locating the source of bleeding in the upper GI tract. ${ }^{9}$ According to the clinical guidelines established by the American College of Physicians, patients who are acutely bleeding should have an upper endoscopy within 24 hours. A metaanalysis comparing patients who had an EGD within the first 12 hours compared to those between 12 to 24 hours found no difference in rebleeding, surgery, or mortality. ${ }^{10}$ Endoscopy findings are described according to the Forrest's classification and can be used to determine the risk of rebleeding of a peptic ulcer and influence treatment decisions.

The risks of EGD include aspiration, perforation, or increased bleeding following therapeutic intervention. A recent study found that endoscopy should not be delayed for low hematocrit or elevated International Normalized Ratio (INR). These studies showed that patients with a hematocrit less than 30 did not have an increased risk of cardiac related events compared to patients with a hematocrit greater than 30 and patients with an INR between 1.3 and 2.7 did not have an increased risk of bleeding following endoscopy. ${ }^{12,13}$ If the endoscopy is nondiagnostic, secondary to blood obstructing, according to the physician's view, it should be repeated.

Patients, with negative upper and lower endoscopies, may be having their small intestine evaluated by capsule endoscopy. Bleeding, originating from the small intestine, accounts for only about $5 \%$ of all GI bleeds. Capsule endoscopy should be used with caution in patients with inflammatory bowel disease, malignancy, or recent bowel anastomoses for risk of perforation. $^{14}$

Computed tomography angiography (CTA): CTA provides a noninvasive and accurate method to determine the location and etiology of a GI hemorrhage. Based on a recent metaanalysis the sensitivity and specificity for CTA diagnosing a GI hemorrhage is 89 and $85 \%$, respectively. CTA can detect bleeding at a rate of $0.5 \mathrm{~mL} / \mathrm{min}$. A triphasic approach consisting of a precontrast phase (to prevent a false positive from pre-existing radiopaque material in the bowel), followed by an arterial phase (to detect active extravasation), and finally, a venous phase scan to assess for progression of contrast extravasation is currently recommended. Oral contrast should not be given because it can obscure the view of extravasation of intravascular contrast. ${ }^{15}$

Catheter angiography: catheter angiography can detect bleeding rates at 0.5 to $1 \mathrm{~mL} / \mathrm{min}$. The sensitivity for diagnosis of GI bleeding with the catheter angiography is 42 to $86 \%$ and specificity is close to $100 \%$. The advantage of catheter angiography is that a vessel can be embolized at the same time, as the procedure if there is active extravasation of the contrast. Unlike the CTA, it has the risk of access-site hematoma, pseudoaneurysm, or arterial dissection. Both CTA and catheter angiography have the risk of contrast induced nephropathy and allergic reaction. ${ }^{15}$

\section{Therapeutic Options}

Initial evaluation of a patient with UGIB involves history, physical examination, and rapid assessment of their hemodynamic status to determine the severity of bleeding. Additionally, two large bore intravenous catheters for fluids and blood should be inserted and a Foley's catheter for closer urine output monitor to guide resuscitation may be considered. Blood transfusion should be considered for patients who are hemodynamically unstable or who have hemoglobin less than $7 \mathrm{~g} / \mathrm{dL}$. Patients taking anticoagulation and/or antiplatelet medications should be considered for reversal if clinically appropriate.

Acid suppression should be started with a PPI. If the bleeding is suspected to be variceal in origin, a continuous intravenous infusion of octreotide should be used to increase splanchnic vasoconstriction and decrease portal flow. Cirrhotic patients with UGIB should receive antibiotics, as 20 to $50 \%$ have been shown to develop infections, such as spontaneous bacterial peritonitis. Endoscopy is the diagnostic and therapeutic modality of choice.

For variceal bleeding, endoscopic varix ligation or sclerotherapy can be performed. Balloon tamponade may also be considered. The balloon may only be kept inflated for a maximum of 24 hours, given risk for esophageal necrosis, with periodic deflation in every 12 hours to check for bleeding. Transjugular intrahepatic portosystemic shunt (TIPS) procedure is an additional option for variceal bleeding that is resistant to endoscopic management.

Most bleeding stops spontaneously or can be controlled endoscopically. Recurrent bleeding should be investigated 
with a repeat EGD. For the 7 to $16 \%$ of cases that have persistent or recurrent bleeding and are high risk for surgery due to cardiac disease, celiac axis and superior mesenteric angiography may be performed with selective transcatheter embolization. Surgical options include oversewing or resecting a bleeding gastric ulcer followed by delayed EGD in 6 to 8 weeks to ensure healing or biopsy to rule out malignancy. Bleeding duodenal ulcers may be managed with oversewing, pyloroplasty, and truncal vagotomy or PPI therapy postoperatively.

Peptic ulcer disease is responsible for $60 \%$ of UGIB. Approximately, $85 \%$ of peptic ulcers are caused by $H$.pylori, with most others caused by nonsteroidal anti-inflammatory drugs (NSAIDS). In the United States, duodenal ulcers are more common than gastric ulcers. Triple therapy with a PPI and the antibiotics amoxicillin or metronidazole and clarithromycin for 2 weeks is the first line therapy for $H$. pylori eradication. Patients are then tested for eradication of $H$. pylori by a urea breath test, endoscopy, or stool antigen 1 month after completion of treatment.

\section{Conclusion}

In conclusion, UGIB is a common problem seen in the emergency room. Early diagnosis and a prompt upper endoscopy (within 24 hours) allow for the best outcome of the patient. Medical therapy may be supplemented with surgery or angiography depending on the patient's condition.

Conflict of Interest

None declared.

\section{References}

1 van Leerdam ME. Epidemiology of acute upper gastrointestinal bleeding. Best Pract Res Clin Gastroenterol 2008;22(02):209-224

2 Holster IL, Kuipers EJ. Management of acute nonvariceal upper gastrointestinal bleeding: current policies and future perspectives. World J Gastroenterol 2012;18(11):1202-1207
3 Dhere T. Chapter 22: Acute Gastrointestinal Bleeding. In: Sitaraman and Friedman's Essentials of Gastroenterology, 2nd ed. John Wiley \& Sons Ltd.; 2018 341-358

4 Gralnek IM, Dumonceau J-M, Kuipers EJ, et al. Diagnosis and management of nonvariceal upper gastrointestinal hemorrhage: European Society of Gastrointestinal Endoscopy (ESGE) Guideline. Endoscopy 2015;47(10):a1-a46

5 Laine L, Jensen DM. Management of patients with ulcer bleeding. Am J Gastroenterol 2012;107(03):345-360, quiz 361

6 Sittichanbuncha Y, Senasu S, Thongkrau T, Keeratikasikorn C, Sawanyawisuth K. How to differentiate sites of gastrointestinal bleeding in patients with hematochezia by using clinical factors? Gastroenterol Res Pract 2013;2013:265076

7 Tomizawa M, Shinozaki F, Hasegawa R, et al. Patient characteristics with high or low blood urea nitrogen in upper gastrointestinal bleeding. World J Gastroenterol 2015;21(24):7500-7505.

8 Pallin DJ, Saltzman JR. Is nasogastric tube lavage in patients with acute upper GI bleeding indicated or antiquated? Gastrointest Endosc 2011;74(05):981-984. Web. 26 Aug. 2016

9 Kim BSM, Li BT, Engel A, et al. Diagnosis of gastrointestinal bleeding: a practical guide for clinicians. World J Gastrointest Pathophysiol 2014;5(04):467-478. Web. 26 Aug. 2016

10 Barkun AN, Bardou M, Kuipers EJ, et al; International Consensus Upper Gastrointestinal Bleeding Conference Group. International consensus recommendations on the management of patients with nonvariceal upper gastrointestinal bleeding. Ann Intern Med 2010;152(02):101-113.

11 Bruno J. Intravenous pantoprazole (Protonix). Available at: http:// www.clevelandclinicmeded.com/medicalpubs/pharmacy/septoct2003/pantoprazole.htm. Accessed May 06, 2019

12 Balderas V, Bhore R, Lara LF, Spesivtseva J, Rockey DC. The hematocrit level in upper gastrointestinal hemorrhage: safety of endoscopy and outcomes. Am J Med 2011;124(10):970-976 Web. 26 Aug. 2016

13 Wolf AT, Wasan SK, Saltzman JR. Impact of anticoagulation on rebleeding following endoscopic therapy for nonvariceal upper gastrointestinal hemorrhage. Am J Gastroenterol 2007;102(02): 290-296. Web. 26 Aug. 2016

14 ACG. Small bowel bleeding. Available at: https://gi.org/topics/ small-bowel-bleeding/. Accessed May 06, 2019

15 Jaskolka JD, Binkhamis S, Prabhudesai V, Chawla TP. Acute gastrointestinal hemorrhage: radiologic diagnosis and management. Can Assoc Radiol J 2013;64(02):90-100. Web. 26 Aug. 2016 